\title{
Radio Sources as Probes of Distant Clusters
}

\author{
Joanne Baker
}

Astrophysics Department, Keble Road, Oxford, OX2 OBA, UK

Jordi Barr

Astrophysics Department, Keble Road, Oxford, OX2 OBA, UK

\author{
Malcolm Bremer \\ Physics, University of Bristol, Tyndall Avenue, Bristol BS8 1TL, UK
}

\begin{abstract}
Powerful radio sources are efficient probes of dense regions throughout the universe, including clusters of galaxies. The status of cluster searches around radio sources is reviewed, including new evidence that cluster environments affect radio morphology.
\end{abstract}

\section{Introduction}

Powerful active galactic nuclei (AGN) - including radio sources - are efficient tracers of dense regions in the universe. The massive ellipticals that host them should be sited in high-density peaks in the galaxy distribution, including clusters. Galaxy mergers in dense environments may also explain the triggering and fueling of the AGN.

Visible out to the highest redshifts, luminous AGN signpost the first forming structures. Counting distant clusters constrains the amount of matter in the universe, $\Omega_{m}$, that ultimately controls the rate at which massive systems collapse (Bahcall \& Fan 1998). Detecting even small numbers of massive clusters at high redshift implies $\Omega_{m}$ is low, less than one.

It is much more efficient to use AGN to locate distant clusters than to carry out a blind survey, although both ways have different biases. Galaxies and intra-cluster gas rapidly become faint at great distances, and are swamped by foreground objects. So blind surveys are difficult beyond $z \sim 1$.

Although both types may live in clusters, radio-loud AGN have many distinct advantages over radio-quiet ones. Dust does not dim the radio emission, whose intrinsic jet power and orientation can be inferred directly. Extended radio lobes also sample the surrounding gas, and intra-cluster medium (ICM) (e.g. Blanton et al. 2003).

McLure \& Dunlop (2001) argue that powerful radio sources at high redshift trace the progenitors of rich clusters today. This follows from the similar space density of powerful radio sources at $z \sim 2.5$ and Abell clusters locally, implying virtually all nearby clusters hosted a powerful radio galaxy in the past. In addition to having massive hosts, powerful (Jy) radio sources have massive central 
black holes $\left(M_{b h} \sim 10^{9} M_{\odot}\right)$, and so are key targets for investigating galaxy and black hole formation and evolution.

More numerous weak ( $\mathrm{mJy}$ ) radio sources can also be mapped on the sky to reveal large galaxy structures, including clusters and superclusters (e.g. Cotter et al. 2002; Brand et al. 2003).

To date, many clusters have been identified around radio sources, including systems at the highest redshifts $(z>4)$. But the wider question of how cluster environments affect AGN and their elliptical hosts remains. Also, it is not obvious how to link the $z>2$ 'protoclusters' with lower redshift clusters and AGN environments.

\section{Clusters around radio sources at $z>2$}

Galaxy overdensities have been reported around a handful of high-redshift radio galaxies, at $z=2-5$. These so-called 'protoclusters' comprise mainly overdensities of tens of star-forming galaxies, visible in Ly $\alpha$ emission (e.g. Venemans et al. 2002), ultraviolet spectral breaks (e.g. Lacy \& Rawlings 1996), or sub-millimeter emission (e.g. Ivison et al. 2000; Smail et al. 2003).

An excess of X-ray sources, but no diffuse cluster gas component, has been seen in one $z=2.2$ field (Carilli et al. 2002).

Typically, the high-redshift radio galaxies found in clusters have unusually clumpy hosts and small, asymmetric radio sources (e.g. Pentericci et al. 1997; 1998).

\section{Clusters around radio sources at $z<2$}

AGN environments have been surveyed systematically out to $z=0.7$, but less so beyond this. Most cluster searches around moderate redshift AGN simply count galaxies within some radius of the AGN. Large studies have characterized AGN environments in this way (Yee \& Green 1987; Ellingson et al. 1991; Hill \& Lilly 1991; Wold et al. 2000; Hall et al. 1998, 2001), and individual rich fields have been identified out to $z \sim 2$ (Sanchez \& Gonzalez-Serrano 1999; Chapman, McCarthy \& Persson 2000).

Incorporating simple color information — selecting extremely red objects around AGN - is one way to alleviate foreground confusion in fields at $z \sim 1$ (e.g. Stern et al. 2003).

Diffuse cluster ICM is detectable around powerful radio sources out to $z \sim 1$ (e.g. Crawford \& Fabian 2003; but see Donahue, Daly \& Horner 2003) but is generally too faint to see at higher redshifts, where microwave background photons scattered by the radio lobes may dominate (e.g. Fabian et al. 2003)

In summary, AGN inhabit a range of environments, mostly of moderate richness (Abell class 0 ) but sometimes very rich (Abell class 2 or 3 ). Relatively few of these clusters have had their masses established by X-ray detections of ICM and/or weak lensing measurements (e.g. Wold et al. 2002; Crawford \& Fabian 2003).

Although early studies proposed evolution in quasar environments from $z=0.7$ to the present day (Yee \& Green 1987; Ellingson et al. 1991; Hill \& 


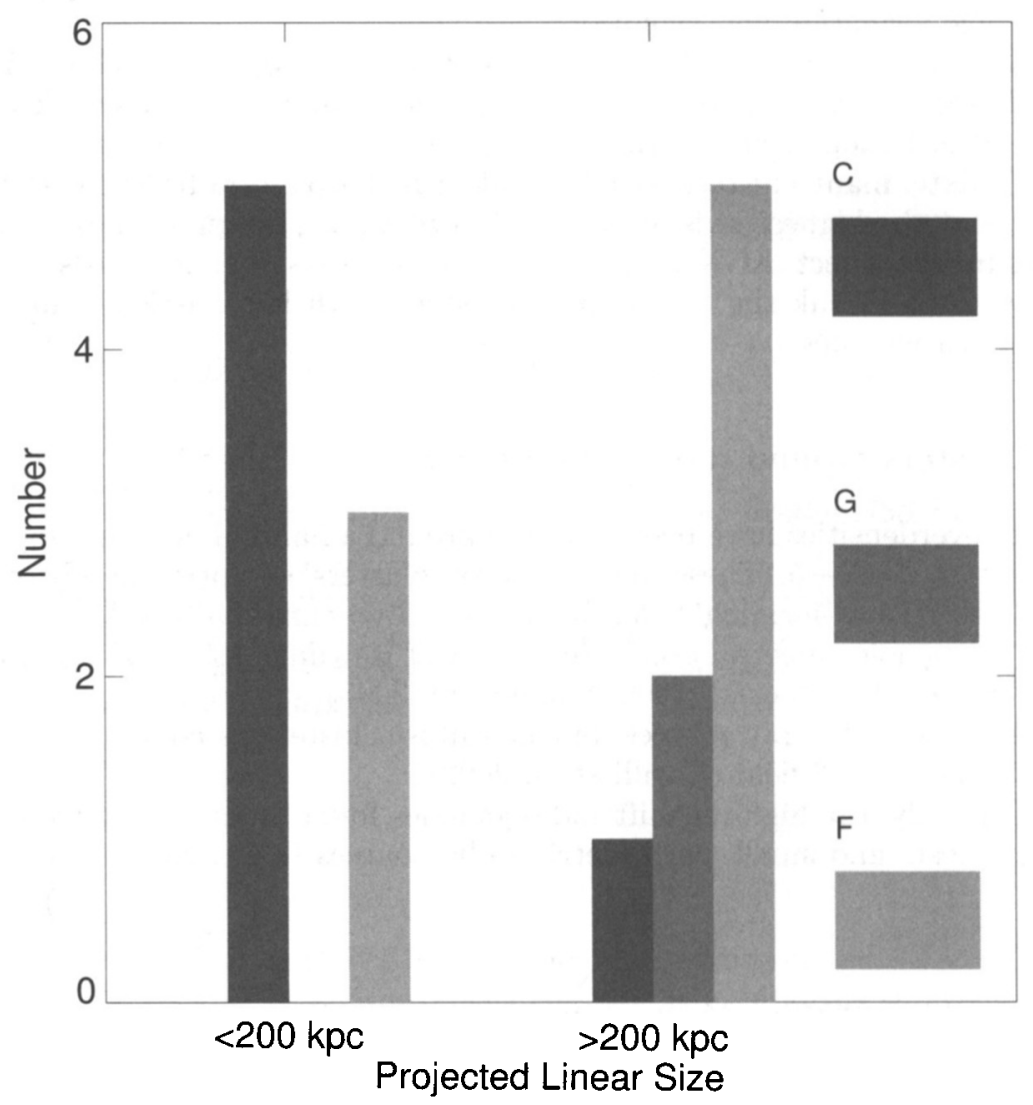

Figure 1. Bar chart showing numbers of radio sources in clusters (c), groups (g) and field (f) environments as a function of radio linear size (D) split at 200 kpc. See Barr et al. (2003).

Lilly, 1981), evidence that environment correlates strongly with AGN properties is weak (Wold et al. 2000).

There is a clear need for systematic studies at $z>0.7$ to push into the epoch where clusters are still coalescing from sub-clumps.

\section{A new survey of quasars at $z \sim 1$}

Barr et al. (2003) imaged the fields of 21 steep-spectrum powerful radio quasars with $0.6<z<1.1$, drawn from a complete redshift-limited subset of the Molonglo Quasar Sample (Kapahi et al. 1998). The fields were imaged in at least two optical filters chosen to straddle the redshifted $4000 \AA$ break feature, selecting the passively-evolving elliptical galaxies that populate cluster cores. 
Some fields were also targeted with narrow-band (FWHM $7 \AA$ ) imaging around the wavelength of redshifted [O II] emission line with the TAURUS Tunable Filter (TTF) at the AAT. This traces strongly star-forming galaxies at the quasar redshift.

Galaxy clustering was measured in two ways (i) by counting galaxies around the quasar, in the same way as previous $z<0.7$ studies, and (ii) counting only red galaxies with the colors expected for passively-evolving, old stellar populations at the quasar redshift.

Red galaxy overdensities were seen in $8 / 21$ fields. Foreground systems (overdensities with the wrong colors) were spotted in two cases, illustrating the weakness of single filter counting statistics. The overdensity centroids lay typically within $10^{\prime \prime}-20^{\prime \prime}$ of the quasar - but not on it - and extended over typically $100^{\prime \prime}$, being frequently elongated.

Looking at the radio morphologies, small (linear size $D<200 \mathrm{kpc}$ ), and asymmetric, radio sources were most often located in clusters $(5 / 8$ small sources compared with $1 / 8$ larger sources in clusters).

In the 6 fields targeted with TTF, 47 emission-line galaxy candidates were detected, with star formation rates $1-100 M_{\odot} \mathrm{yr}^{-1}$ and very faint $(I(A B)>23)$ continua. Emission-line galaxy candidates cluster around the quasars, but less strongly than the red galaxies, suggesting the morphology-density relation is in place at $z \sim 1$.

\section{Conclusion}

Powerful radio sources - especially small, asymmetric ones - are often located in clusters of galaxies that are seen out to the highest redshifts.

At redshifts $z>0.7$, they are best located using multicolor and/or narrowband techniques, along with $\mathrm{X}$-ray and sub-millimeter observations.

More work is needed on a systematic basis to measure the masses of these systems (e.g. diffuse X-rays, spectroscopy and weak lensing techniques) and to understand links between AGN and their environments.

Acknowledgments. JCB acknowledges support from a Royal Society University Research Fellowship.

\section{References}

Bahcall, N, Fan X. 1998, ApJ, 504, 1

Barr, J., et al. 2003, MNRAS, 346, 229

Blanton, L., et al., 2003, AJ, 125, 1635

Brand, K, et al. 2003., MNRAS, 344, 283

Carilli, C., et al. 2002, ApJ, 567, 781

Chapman, S., McCarthy P., Persson S. 2000, AJ, 120, 1612

Cotter, G., et al. 2002, MNRAS, 331, 1

Crawford, C., Fabian, A. 2003, MNRAS, 339, 1163

Donahue, M., Daly R., Horner D. 2003, ApJ, 584, 643

Ellingson, E., Yee, H., Green R. 1991, ApJ, 371, 49

Fabian A., Sanders, J., Crawford C., Ettori, S. 2003, MNRAS, 341, 729 
Hall, P., et al. 2001, AJ, 121, 1840

Hall, P., Green, R. 1998, ApJ, 507, 558

Hill, G., Lilly, S. 1991, ApJ, 367, 1

Ivison, R., et al. 2000, ApJ, 542, 27

Kapahi, V., et al. 1998, ApJS, 118, 327

Lacy M., Rawlings S. 1996, MNRAS, 280, 888

McLure, R., Dunlop, J. 2001, MNRAS, 327, 199

Pentericci, L., et al. 1998, ApJ, 504, 139

Pentericci, L., et al. 1997, å, 326, 580

Sanchez, S., Gonzalez-Serrano, J. 1999, å, 352, 383

Smail, I., et al. 2003, ApJ, 583, 551

Stern, D., Holden, B., Stanford, S., Spinrad, H. 2003, AJ, 125, 2759

Venemans, B., et al. 2002, ApJ, 569, L11

Wold, M., et al. 2002, MNRAS, 335, 1017

Wold, M., Lacy, M., Lilje, P., Serjeant, S. 2000, MNRAS, 316, 267

Yee, H., Green, R. 1987, ApJ, 319, 28 